\title{
Investigating site-specific interactions and probing their role in modifying the acid-strength in framework architectures
}

\author{
Matthew E. Potter ${ }^{a^{*}}$, Danni Sun ${ }^{a}$, Enrica Gianotti ${ }^{\mathrm{b}}$, Maela Manzolic and Robert Raja ${ }^{\mathrm{a} *}$ \\ Received (in $X X X, X X X) X t h X X X X X X X X X 20 X X$, Accepted Xth XXXXXXXXX 20XX \\ DOI: $10.1039 / \mathrm{b} 000000 x$
}

The ability to adroitly tailor acid-strength using specificallyengineered bimetallic nanoporous materials has been investigated with a view to exploiting their potential in solidacid catalysed transformations. Further, it has been ${ }_{10}$ demonstrated that through site-specific interactions, extraframework zinc ions can suitably modify the acidity of Bronsted acid sites, to stimulate diverse catalytic responses, when combined with isomorphously-substituted framework metal cations within porous architectures, for the Beckmann 15 rearrangement of cyclohexanone oxime and in the isopropylation of benzene.

\section{Introduction}

The continued demand for the development of sustainable chemical processes has prompted the discovery and design of 20 novel catalytic materials that operate in an environmentally benign fashion, in order to meet the stringent requirements of clean technology and energy efficiency. ${ }^{[1-3]}$ Understanding the nature of the active site at the molecular level is therefore a fundamental prerequisite, for facilitating structure-property 25 correlations, that enable the design of catalytically active entities for enhancing rates and selectivity. ${ }^{[4,5]}$

It is well-known that a diverse array of synthetic approaches ${ }^{[6-}$ ${ }^{10]}$ can be adopted for heterogenizing a wide-variety of molecular precursors on high-area supports for generating multimetallic 30 metal nanoparticle catalysts that lead to enhanced catalytic turnovers. $^{[11-13]}$ Recently, ${ }^{[4,14,15]}$ the simultaneous isomorphous substitution of multimetallic transition-metal ions into the framework of porous architectures has resulted in considerable enhancements in catalytic synergy in selective oxidation 35 reactions. The implementation and execution of such a design strategy often requires precise dexterity in the synthesis procedure, manipulation and control of the pore dimensions of the framework architecture, choice of dopant metal precursors, coupled with a detailed elucidation of the nature of the active site 40 by employing advanced spectroscopic and structural characterisation methods. ${ }^{[16-21]}$

The incorporation of multimetallic species has often led to the modification of intrinsic nature of the active site that has subsequently led to enhancements in catalytic efficiencies in 45 nanoparticle-based catalysts and bulk oxides. ${ }^{[11,22]}$ Alloying suitable oxophiles in nanoclusters-based compositions facilitates the generation of isolated active centres, resulting in a significant improvement in catalytic turnover. It has been shown that the oxophile does not play an implicit role in the catalytic process; ${ }_{50}$ but helps to securely anchor the nanoparticle entities to the silica support, through the pendant silanol groups that are present on the high surface area of the support. ${ }^{[5]}$

This simultaneous incorporation of bimetallic cations in microporous aluminophosphate (AlPO) frameworks offers 55 adequate scope for facilitating bifunctional catalytic transformations ${ }^{[23]}$ or afford alternate mechanistic pathways by providing specific binding sites for the coordination of substrate and oxidant molecules. These materials have the ability to isomorphously incorporate small quantities of metal dopants into 60 their porous architecture, forming isolated active sites (Figure 1). ${ }^{[24-25]}$ Through a judicious choice of metal precursors, one can bestow distinct catalytic properties to the porous matrix, thereby facilitating redox and acid-catalysed transformations in a singlestep. ${ }^{[23]}$ Similarly, if the metal loadings are suitably optimised, it 65 is possible to adapt synthetic procedures to incorporate a variety of metal-ions into the same framework, ${ }^{[26]}$ creating complementary, yet distinct, active sites. ${ }^{[11,14,23]}$

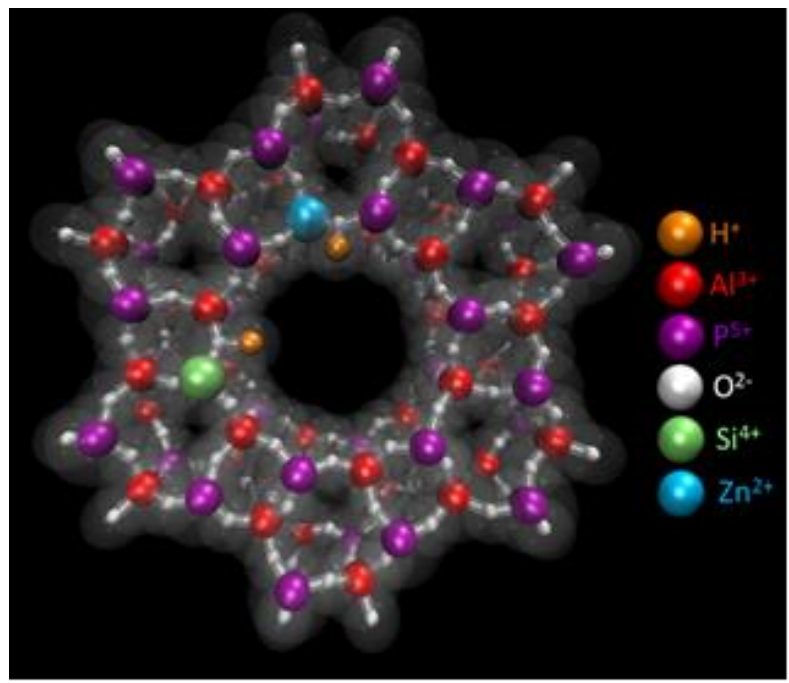

Figure 1 Schematic of a bimetallic $\mathrm{Zn}^{2+} \mathrm{Si}^{4+} \mathrm{AlPO}-5$ system

70 Current research into designing bimetallic molecular sieves for selective oxidations has revealed $^{[4,14,15]}$ that multi-metallic incorporation facilitates site-specific interactions, thereby extending versatility of this approach. Many recent examples 
have shown that new synthetic directions involving bimetallic catalysts lead to improved catalytic efficiencies for hydrocarbon transformations by exploiting inherent synergistic enhancements between different metal species. ${ }^{[4,13-15,27,28]}$ We have recently 5 extended $^{[14,15]}$ the synthetic strategy to encompass a range of transition-metal and heavy-metal bimetallic analogues, where the proximity of different heteroatom dopants could be precisely controlled. Further, by positioning the two metal species in sufficiently close proximity, the coordination geometry of the 10 individual species can be modified, facilitating remarkable increases in catalytic performance. ${ }^{[4]}$

From an application perspective, ${ }^{[29]}$ we have demonstrated earlier that incorporating two distinct acid sites within the same nanoporous catalyst, ${ }^{[29]}$ modifies the local structural environment 15 around the active site and improves the catalytic efficiency of the material in the dehydration of ethanol. In this work, we demonstrate how $\mathrm{Zn}^{2+}$ ions, introduced into a microporous molecular sieve, are able to adroitly tailor the nature of both $\mathrm{Mg}^{2+}$ and $\mathrm{Si}^{4+}$ sites, that reside in the immediate vicinity of the 20 framework. It is widely known that $\mathrm{Zn}$ acts as a modifier in certain chemical transformations, ${ }^{[30]}$ and, given the comparable ionic size and valency to $\mathrm{Mg}$, it was hoped that $\mathrm{Zn}$ would be a good candidate for prompting synergistic interactions. In this work, we compare the behaviour of $\mathrm{Zn}$-containing and $\mathrm{Zn}$-free 25 catalysts for two acid-catalysed transformations: the vapourphase Beckmann rearrangement of cyclohexanone oxime and the isopropylation of benzene (Scheme 1), with a view to understanding the nature of the acid sites and the influence of zinc.

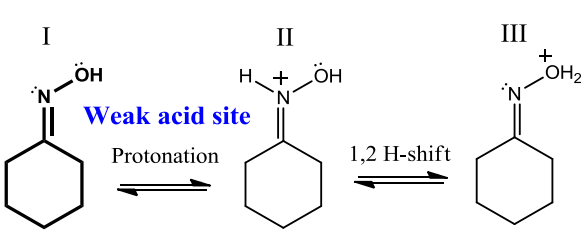

A
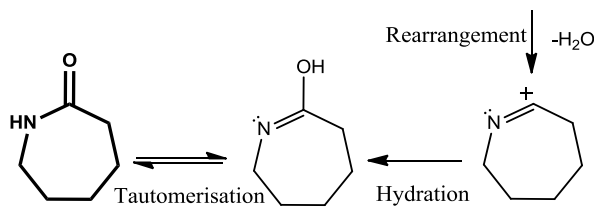

VI

$\mathrm{V}$
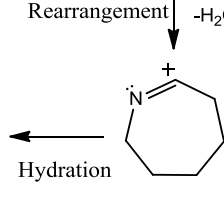

30

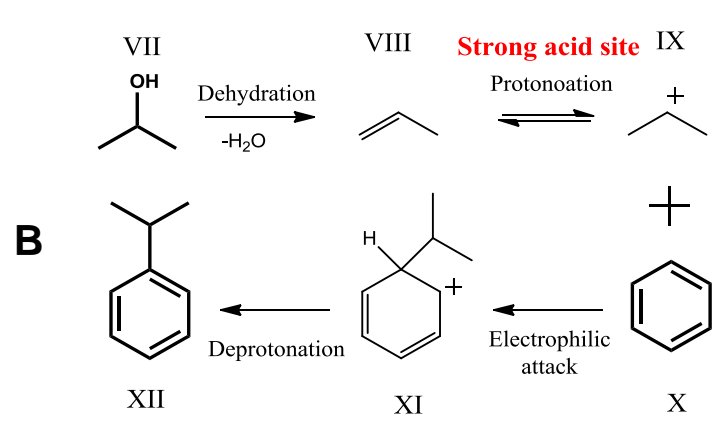

Scheme 1 Schematic detailing the contrasting acid sites required for the Beckmann rearrangement of cyclohexanone oxime to \&-caprolactam (A) and the isopropylation of benzene to cumene (B), highlighting the rate-

35 determining steps in terms of the 1,2 H-shift (in A) and electrophilic attack (in B) respectively.

These examples (Scheme 1) were selected, both for their significance in the polymer and fine-chemical industries, and also for their preference in involving contrasting Brønsted acid 40 species. The production of $\varepsilon$-caprolactam from the Beckmann rearrangement $(\mathbf{A})$ is strongly dependent on the presence of weak Brønsted acid sites; ${ }^{[31]}$ with stronger acid sites favouring byproducts and coking. ${ }^{[32]}$ In stark contrast, the isopropylation of benzene requires the involvement of strong Brønsted acid sites, in 45 order to activate isopropanol. ${ }^{[33]}$ By unifying the results of the complementary catalytic tests, and by employing in situ FTIR spectroscopy in combination with suitable probe molecules that would reveal the nature and strength of the acid sites, the influence of zinc (incorporation) could be suitably rationalised. It 50 was hoped that the above studies would facilitate structureproperty correlations to be established, which could lead to the design and fabrication of specific active sites for targeted acidcatalysed transformations.

\section{Results and discussions}

\section{${ }_{55}$ Textural characterisation}

The effect of incorporating zinc into the microporous architecture was investigated using a range of physic-chemical characterisation techniques. This study aimed to draw structureproperty correlations by probing the precise nature of the ${ }_{60}$ bimetallic active species (acid sites), whilst also considering the intrinsic textural properties of the nanoporous framework. The incorporation of metal dopants into the AlPO framework did not reveal any structural and phase imperfections and the intended crystalline AFI framework was preserved (Figure 2).

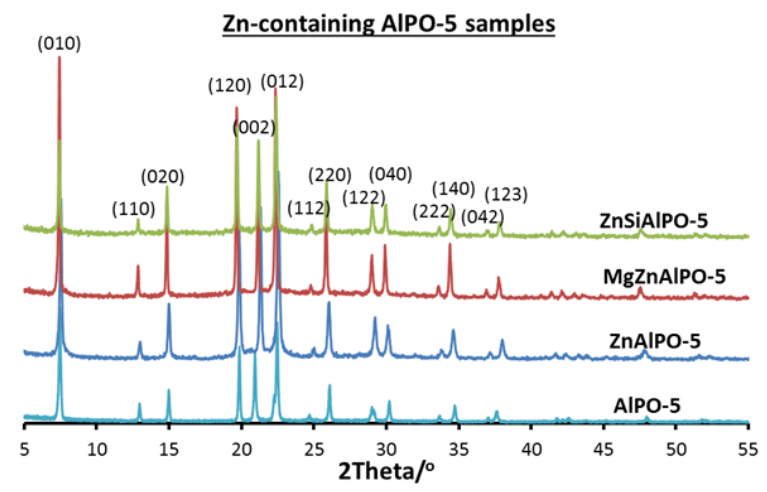

${ }_{65}$ Figure 2 Powder X-ray diffraction patterns of Zn doped AlPO-5 catalysts.

Table 1 Summary of textural characterisation

\begin{tabular}{lllll}
\hline System & \multicolumn{2}{c}{ Optimized XRD } & Particle & BET \\
& parameters for P6cc & size/nm & SSA $/ \mathrm{m}^{2} \mathrm{~g}^{-1}$ \\
& $\mathrm{a} / \AA$ & $\mathrm{c} / \AA$ & & \\
\hline AlPO-5 & 13.69 & 8.43 & 56.5 & 295.1 \\
ZnAlPO-5 & 13.68 & 8.34 & 55.5 & 165.4 \\
MgAlPO-5 & 13.71 & 8.40 & 55.5 & 193.3 \\
MgZnAlPO-5 & 13.80 & 8.41 & 66.3 & 283.3 \\
SiAlPO-5 & 13.70 & 8.39 & 54.8 & 181.9 \\
ZnSiAlPO-5 & 13.76 & 8.40 & 64.2 & 236.2 \\
\hline
\end{tabular}

70 The structural parameters were refined (Reitveld) and further quantified, through unit cell parameters and particle size calculations, all of which showed only a subtle deviation from the 

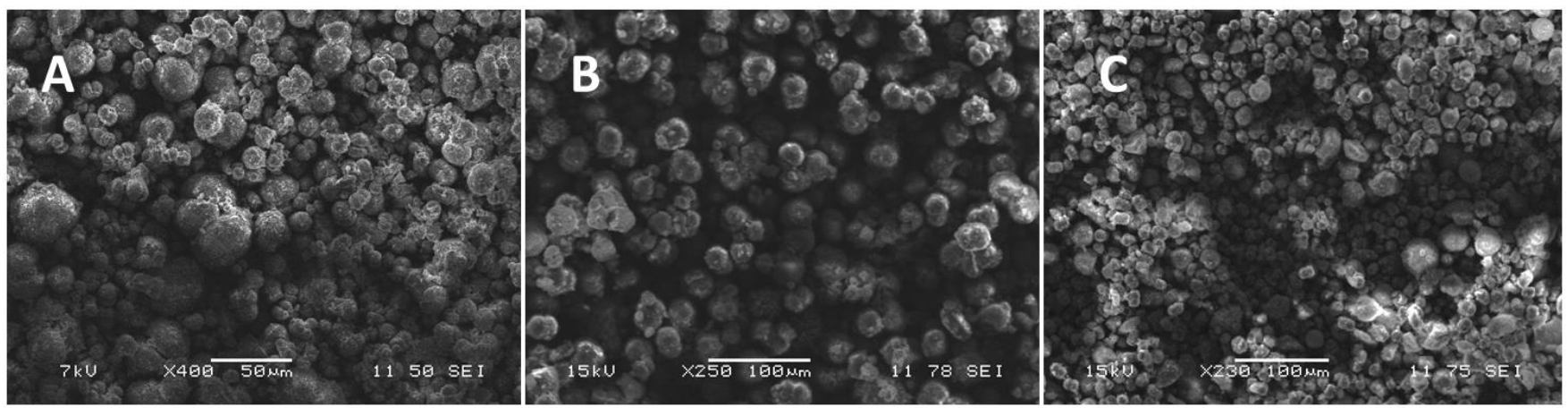

Figure 3 SEM images of ZnAlPO-5 (A), MgZnAlPO-5 (B) and ZnSiAlPO-5 (C).

undoped-phase; confirming the extent of heteroatom incorporation that was achieved (Table 1). In all cases the maximum derivation from the undoped crystalline parameters was less than $1 \%$, in agreement with the low quantities of 5 heteroatoms doped into the framework (Table S2). This variance in the unit cell parameters facilitates a shift in peak position from that of AlPO-5. However, closer inspection of the XRD patterns in the $30-55^{\circ} 2 \theta$ region reveals peaks related to hexagonal $\mathrm{ZnO}$ phase, indicating that a very small proportion of $\mathrm{Zn}$ is present as 10 extra-framework crystalline $\mathrm{ZnO}$ particles, possibly inside the channels (Figure S1). Given the low intensity of the $\mathrm{ZnO}$ peaks, coupled with the low levels of zinc present in the systems, it is not possible to exclude the presence of other $\mathrm{Zn}$ sites. SEM imaging techniques exclusively showed the expected spherical 15 AlPO-5 particles in the region of $10-20 \mu \mathrm{m}$, whilst showing no evidence other phases (Figure 3). The Scherrer equation further reinforces that the particles are of a similar size.

To gain further insights on the location of the zinc ions, specific surface area measurements were employed to compare 20 the bimetallic and monometallic catalysts to that of the undoped AlPO-5 framework $\left(295 \mathrm{~m}^{2} / \mathrm{g}\right.$, Table 1). The greatest variation was seen in the monometallic ZnAlPO-5 system, that revealed a significant decrease in surface area $\left(165 \mathrm{~m}^{2} / \mathrm{g}\right)$, which is indicative of pore obstruction by extra-framework $\mathrm{Zn}$ species. In 25 stark contrast, very little variation was seen in the bimetallic catalysts, confirming the truly porous nature of these materials and their suitability for deployment in catalytic applications.

Initially direct observation of the O-H stretching region (3800$3400 \mathrm{~cm}^{-1}$, Figure S2) showed a band at $3678 \mathrm{~cm}^{-1}$, which is 30 attributable to free $\mathrm{P}-\mathrm{OH}$ defect sites $^{[34]}$ that are ubiquitous in AlPO species. In the Si-containing catalysts, a band at $3745 \mathrm{~cm}^{-1}$ is also present, indicative of available $\mathrm{Si}-\mathrm{OH}$ groups. This band is particularly pronounced in the $\mathrm{ZnSiAlPO}-5$ catalyst, indicating that a greater quantity of these sites are present in this sample; 35 despite keeping the effective Si-loading at the same level with that of its monometallic counterpart, SiAlPO-5. Although the presence of these peaks is perceptible, they only provide partial information on the true nature and strength of the acid sites. Given the high scattering profile of AlPO-5 materials in the 40 stretching region of Brønsted acid sites $\left(3650-3500 \mathrm{~cm}^{-1}\right)$, this kind of strong acid sites cannot be directly detected. Instead, to gain a more detailed understanding of the Brønsted acid sites present in metal-substituted AlPO-5, the use of probe molecules is necessary.

45 A weak base such as CO and a strong base such as 2,6-DMP were employed as contrasting probe molecules to elucidate the strength and relative amounts of the acid sites present in the AlPO-5 samples. $\mathrm{CO}$ was adsorbed at $80 \mathrm{~K}$ to observe its interaction with the $\mathrm{OH}$ groups present in the nanoporous 50 materials. To understand the nature of the acid sites specific focus was placed on the hydrogen-bonded C-O stretching range (2200$2100 \mathrm{~cm}^{-1}$ ), as reported in Figure 4.

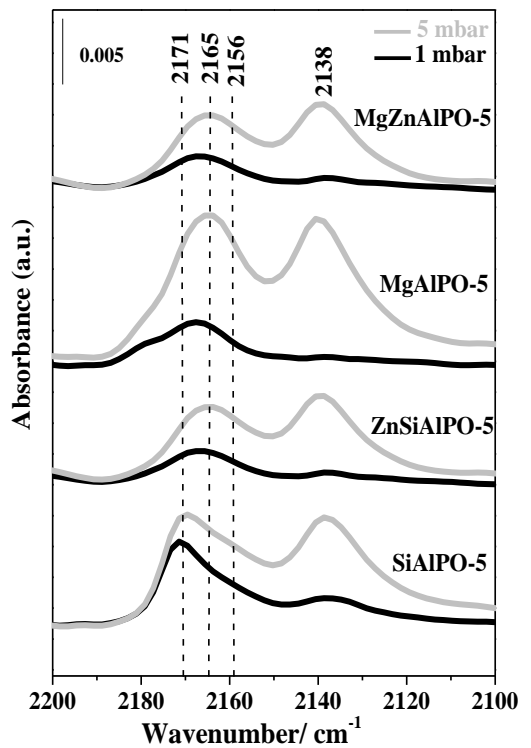

Figure 4 FTIR spectra of $\mathrm{CO}$ adsorbed at $80 \mathrm{~K}$ on calcined SiAlPO-5, ${ }_{55} \mathrm{ZnSiAlPO}-5$, MgAlPO-5 and MgZnAlPO-5; black curve: 1 mbar, grey curve: 5 mbar of $\mathrm{CO}$

In all spectra, the particularly pronounced band at $2138 \mathrm{~cm}^{-1}$ upon the adsorption of 5 mbar of $\mathrm{CO}$, is attributed to liquid-like $\mathrm{CO}{ }^{[35]}$ The band at $2165 \mathrm{~cm}^{-1}$ is assigned to $\mathrm{CO}$ interacting ${ }_{60}$ exclusively with P-OH groups, ${ }^{[36]}$ being the only hydroxyl groups present in the undoped AlPO-5 system (Figure S3). The spectra of the Si-containing samples also shows a shoulder (particularly visible upon adsorption of 5 mbar of $\mathrm{CO}$ ) at ca. $2156 \mathrm{~cm}^{-1}$, which is associated with the interaction of $\mathrm{CO}$ with the pendant surface ${ }_{65}$ silanol groups present on these materials. ${ }^{[37]}$ In addition, a further component at $2171 \mathrm{~cm}^{-1}$, which is assigned to the stretching mode of $\mathrm{CO}$ interacting via $\mathrm{H}$-bond with framework Brønsted acid sites, is also visible. ${ }^{[38-41]}$ The presence of such bands provides a strong indication for framework incorporation of the $\mathrm{Mg}^{2+}$ and ${ }_{70} \mathrm{Si}^{4+}$ ions into the AlPO-5 framework, which leads to the creation of Brønsted acid sites. Moreover, the framework incorporation of $\mathrm{Mg}^{2+}$ ions is also confirmed by the appearance of a shoulder at $2180 \mathrm{~cm}^{-1}$ in the spectra of MgAlPO-5 catalyst, due to the direct 
interaction between framework $\mathrm{Mg}^{2+}$ and $\mathrm{CO}$ molecules. ${ }^{[42]}$

While $\mathrm{CO}$ adsorption is unable to distinguish between Brønsted sites with different acid strengths, the intensity of the signal at $2171 \mathrm{~cm}^{-1}$ is related to the relative quantity of Brønsted 5 acidity in the systems. By comparing the intensity of this region for the MgAlPO-5 and ZnMgAlPO-5 pairing and also of the SiAlPO-5 and ZnSiAlPO-5 pair, it is evident that the inclusion of $\mathrm{Zn}$ reduces the total number of acid sites in the nanoporous frameworks. Given the comparable levels of $\mathrm{Si}$ and $\mathrm{Mg}$ found in 10 the respective systems (Table S2), it is reasonable that zinc species are solely responsible this reduction in total acidity. These findings further emphasise the effect that different dopants can have on the crystallisation process. As the $\mathrm{Mg}^{2+}$ and $\mathrm{Zn}^{2+}$ ions are of similar ionic size, it would be reasonable to assume similar 15 levels of incorporation of these ions, thereby translating to a similar fraction of Brønsted acid sites. The experimental observations, however, accentuate the degree of complexity involved in the kinetics of crystallisation, and show that while $\mathrm{Mg}^{2+}$ ions are readily incorporated into the framework; 20 facilitating the formation of Brønsted acid sites, $\mathrm{Zn}^{2+}$ ions prefer to exist as extra-framework species. This observation is confirmed when comparing the CO adsorbed FTIR spectra of MgAlPO-5, ZnAlPO-5 and AlPO-5 (Figure S3).

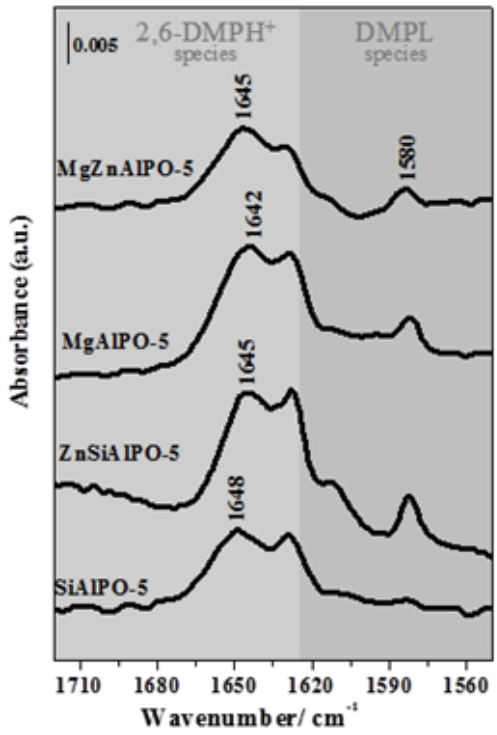

Figure 5 FTIR spectra of outgassed 2,6-DMP at $298 \mathrm{~K}$ on calcined ${ }_{25}$ SiAlPO-5, ZnSiAlPO-5, MgAlPO-5 and MgZnAlPO-5.

Performing adsorption experiments with 2,6-DMP at room temperature allows the effect of bimetallic incorporation to be explored further by clarifying the strength and the nature of the ${ }_{30}$ Brønsted acid sites present in the catalysts. It is known that 2,6DMP yields the protonated species more readily than pyridine, due to its stronger basicity ${ }^{[43]}$ and its weaker affinity for Lewis acid sites, owing to the steric hindrance induced by the methyl groups. ${ }^{[4]}$ The $v_{8 \mathrm{a}}(\mathrm{CC})$ mode is very sensitive and allows the 35 identification of different types of adsorption of the probe molecule on solids. In particular, it is related to protonated species $\left(2,6-\mathrm{DMPH}^{+}\right)$when the $v_{8 \mathrm{a}}$ wavenumber is higher than $1625 \mathrm{~cm}^{-1}$; whereas it corresponds to coordinated or H-bonded species (DMPL) at lower wavenumber. Upon 2,6-DMP
40 adsorption, the position of the $v_{8 \mathrm{a}}(\mathrm{CC})$ band was monitored in the FTIR spectra of the catalysts outgassed at $298 \mathrm{~K}$, in which only the irreversibly adsorbed species are present (Figure 5 \& S4). Moreover, the absorption related to $2,6-\mathrm{DMPH}^{+}$is present in all substituted AlPO-5 samples (1655-1640 $\mathrm{cm}^{-1}$ range).

45 By directly observing the $v_{8 \mathrm{a}}(\mathrm{CC})$ band of adsorbed 2,6-DMP, correlations can be made with the position of this band to both the nature and the strength of acid sites present in the material: the stronger the Brønsted acidity, the lower the position of the band. ${ }^{[45]}$ For all the catalysts used in this study, this band was ${ }_{50}$ found to be in the range of $1642-1650 \mathrm{~cm}^{-1}$, confirming the Brønsted nature of the acid sites. The peak maxima of the diagnostic $v_{8 \mathrm{a}}(\mathrm{CC})$ band is reported in Table 2 \& Figures 5 \& S4.

Table 2 Position of the $v_{8 \mathrm{a}}(\mathrm{CC})$ band in the mono and bimetallic AlPO-5 catalysts

\begin{tabular}{cc}
\hline Catalyst & $v_{8 \mathrm{a}}(\mathrm{CC}) / \mathrm{cm}^{-2}$ \\
\hline MgAlPO-5 & 1642 \\
MgZnAlPO-5 & 1645 \\
& \\
SiAlPO-5 & 1648 \\
ZnSiAlPO-5 & 1645 \\
& \\
ZnAlPO-5 & 1645 \\
AlPO-5 & 1650 \\
\hline
\end{tabular}

55

From the above findings, it can be inferred that the addition of $\mathrm{Zn}$ provokes contrasting behaviour in the various monometallic catalysts. $\mathrm{Mg}^{2+}$ ions are known to induce the strongest Brønsted acid sites in AlPO species; which is concurrent with our findings 60 above, as the MgAlPO-5 catalyst yields the lowest $v_{8 \mathrm{a}}(\mathrm{CC})$ band position $\left(1642 \mathrm{~cm}^{-1}\right)$. The incorporation of zinc into the MgAlPO5 catalyst inherently weakens these acid sites, as noted from the increase of the $v_{8 \mathrm{a}}(\mathrm{CC})$ band $\left(1645 \mathrm{~cm}^{-1}\right)$. The curious influence of the zinc species is further accentuated, as it induces the 65 opposite effect on the $\mathrm{Si}^{4+}$ sites, where the acid site strength is relatively increased, as the $v_{8 \mathrm{a}}$ band is lowered from $1648 \mathrm{~cm}^{-1}$ in SiAlPO-5 to $1645 \mathrm{~cm}^{-1}$ in ZnSiAlPO-5. This rather peculiar behaviour confirms that zinc does not have a uniform effect on active sites that are isomorphously incorporated into AlPO 70 frameworks. On the other hand, it forms extra-framework species capable of having specific and site-selective interactions with different metal cations in the AlPO-5 framework.

\section{Catalysis}

\section{Monometallic systems}

75 The vapour-phase Beckmann rearrangement of cyclohexanone oxime and the isopropylation of benzene reactions were studied not only for their relevance in industry; but as they also provide complementary information on the nature of the active site (see Scheme 1). Given the analogous procedures employed in the 80 syntheses of these catalysts and from the key findings arising from their physico-chemical characterisation under identical conditions, it is reasonable to assume that any variations in catalytic activity are not influenced by the nanoporous architecture. Instead any differences in activity and selectivity are 85 solely due to the individual metal ions present. This is clearly evident from the fact that the framework-substituted $\mathrm{Mg}^{2+}$ ions in 
MgAlPO-5, containing strong Brønsted acid sites as apparent from FTIR data (Figures 4, 5, S4 and Table 2), displays a superior catalytic performance in the isopropylation of benzene (Figure 6B), by possessing the necessary strong acid-sites capable 5 of protonating the propene molecule, thereby forming an electrophile capable of partially breaking the aromaticity of the benzene ring (Scheme 1B). In stark contrast, the SiAlPO-5 catalyst that has a higher fraction of the weaker acid sites, exhibits a superior efficiency for the vapour-phase Beckmann 10 rearrangement of cyclohexanone oxime (Figure 6A), whereas the stronger acid sites in the MgAlPO-5 catalyst hinder the ratedeterming 1,2 $\mathrm{H}$-shift by anchoring the proton to the nitrogen (Scheme 1A). Furthermore, such strong sites embed the $\varepsilon$ caprolactam product to the surface, that results in a decrease in 15 the observed selectivity, through coking and deactivation of the catalyst. ${ }^{[32]}$ The direct correlation between the contrasting catalytic behaviour of the SiAlPO-5 and MgAlPO-5, coupled with the FTIR findings underpinning the nature of the active sites, emphasises the influence of acidity for the complementary 20 catalytic reactions employed in this study (Scheme 1).

By comparing the overall activity of the monometallic catalysts in both processes, it is readily apparent that the ZnAlPO-5 catalyst is far inferior to the Mg- or Si-AlPO-5 analogues (Figure 6A, 6B, S5 \& S6). In accordance with the 25 findings from the XRD and spectroscopic data, the zinc ions are reluctant to undergo isomorphous framework substitution into the AlPO architecture to form Brønsted acid sites. This is further reiterated from the presence of a $\mathrm{ZnO}$ extra-framework species and from the low-intensity of the CO adsorbed bands in the FTIR 30 spectra (Figure S3). This subsequently results in the framework possessing a negligible fraction of isolated $\mathrm{Zn}^{2+}$ sites, with fewer acid sites than the undoped AlPO-5 analogue, rendering the monometallic ZnAlPO-5 system catalytically inactive. The combination of the structural findings, in conjunction with the 35 associated spectroscopic implications that are further reinforced with the catalysis results, overwhelmingly suggests that the $\mathrm{Zn}^{2+}$ ions serve only as catalytically-inert, extra-framework spectator species, in the catalytic process.

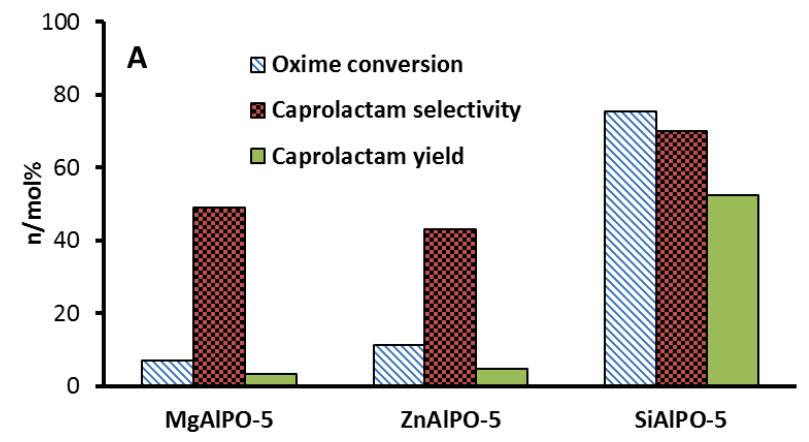

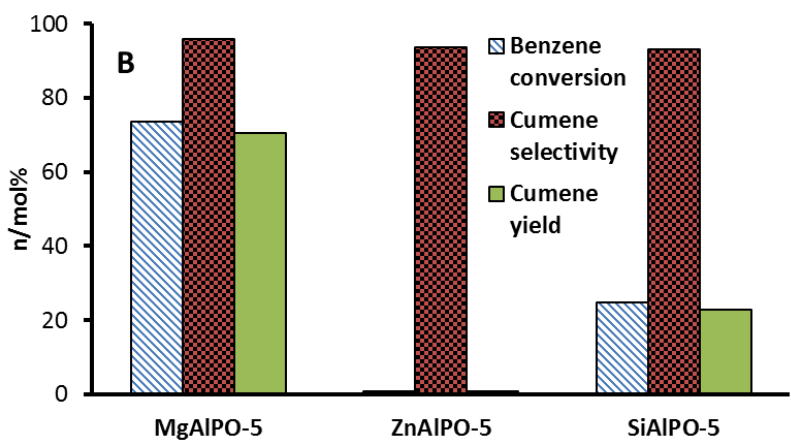

Figure 6 Activity of monometallic catalysts on the vapour-phase Beckmann rearrangement (A) and isopropylation of benzene (B). Conditions: A) WHSV $3.3 \mathrm{hr}^{-1}$, Helium carrier gas flow $20 \mathrm{ml} / \mathrm{min}, 0.3 \mathrm{~g}$ of catalyst, liquid feed $300 \mathrm{~g} / \mathrm{l}$ of cyclohexanone oxime in methanol, 45 temperature $673 \mathrm{~K}$. B) WHSV of $3.5 \mathrm{hr}^{-1}$, Helium carrier gas of 10 $\mathrm{ml} / \mathrm{min}$, feed 6:1 mole ratio of benzene:isopropanol, temperature $523 \mathrm{~K}$.

\section{Bimetallic Catalysts}

The striking spectroscopic differences in the quantity, strength and nature of the active sites, through the incorporation of zinc to 50 form bimetallic catalysts, has been already exemplified through in situ FTIR studies; and these are directly manifested in the nature of their catalytic results. The variance in catalytic behaviour between MgAlPO-5 and MgZnAlPO-5 clearly emphasises the detrimental effects of $\mathrm{Zn}$ in the isopropylation 55 reaction, which significantly retards both the activity and selectivity over a wide-range of temperatures, as illustrated in Figures 7A and S8. Whilst the effect is most pronounced when considering the conversion of benzene, the selectivity to cumene provides further evidence on the reduction in acid strength. The 60 weaker acid sites present in the bimetallic MgZnAlPO-5 catalyst generates a less potent electrophile, which favours attack of the activated cumene aromatic system, over that of the comparatively inert benzene, fostering the formation both the di- and trialkylated products. This observation is further exemplified when ${ }_{65}$ considering the Beckmann data, where MgZnAlPO-5 shows significant improvements over both the monometallic MgAlPO-5 (Figure 7B) and ZnAlPO-5 systems (Figure S5 and S7). Beyond $623 \mathrm{~K}$, the difference in overall activity is conspicuous, as the weaker-acidic sites initiate the isomerisation process. Despite $70 \mathrm{MgAlPO}-5$ possessing a greater fraction of Brønsted acid sites, the MgZnAlPO-5 is more proficient for this reaction, further illustrating that it is not exclusively the overall quantity, but the nature and strength of the acid site, which is pivotal for efficiently promoting these acid-catalysed hydrocarbon transformations.

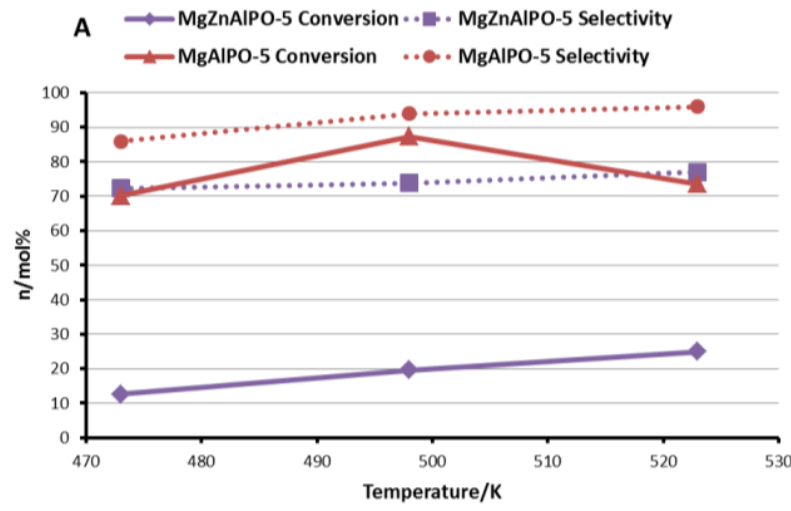




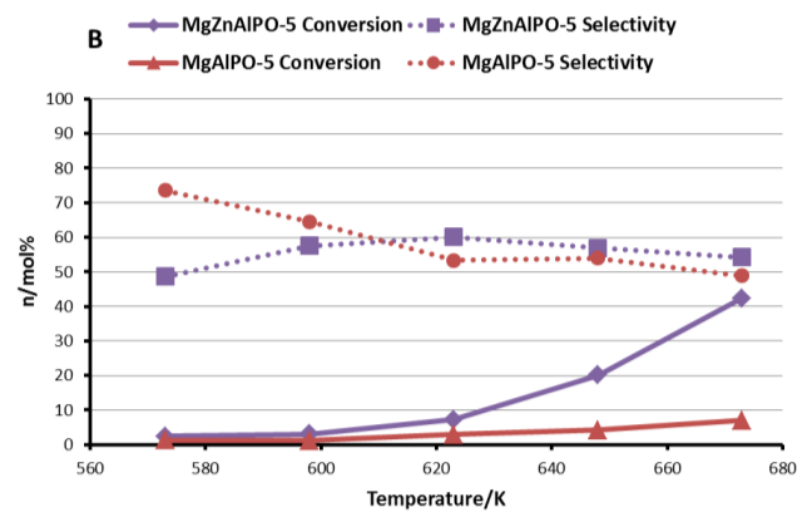

Figure 7 Activity of Mg-containing catalysts in the isopropylation of benzene (A) and vapour-phase Beckmann Rearrangement (B).

Conditions: A) WHSV of $3.5 \mathrm{hr}^{-1}$, Helium carrier gas of $10 \mathrm{ml} / \mathrm{min}$, feed ${ }_{5}$ 6:1 mole ratio of benzene:isopropanol, temperature as shown. B) WHSV $3.3 \mathrm{hr}^{-1}$, Helium carrier gas flow $20 \mathrm{ml} / \mathrm{min}, 0.3 \mathrm{~g}$ of catalyst, liquid feed $300 \mathrm{~g} / \mathrm{l}$ of cyclohexanone oxime in methanol, temperature as shown.

Similarly the distinctions in catalytic findings (Figure 8) between the bimetallic $\mathrm{ZnSiAlPO}-5$ and the $\mathrm{Zn}$-free 10 monometallic SiAlPO-5 catalysts are evident, and these are again in accordance with the nature of the acid sites that are present, as illustrated by their associated spectroscopic findings. The negligible decrease in the $\varepsilon$-caprolactam yield (beyond $498 \mathrm{~K}$ ) can be attributed to the in situ addition of a small amount of zinc 15 to the SiAlPO-5 system (Figure 8A and S9), notwithstanding the fact that this leads to the formation of crystalline $\mathrm{ZnO}$ particles. This modification of the weak acid sites present in the SiAlPO-5 catalyst is also responsible for a marked improvement in the formation of cumene with the ZnSiAlPO-5 catalyst (Figure 8B 20 and S10). The significant alterations in catalytic activity are a result of an increase in acid-site strength, facilitated by the presence of $\mathrm{Zn}^{2+}$ ions within the microporous system.

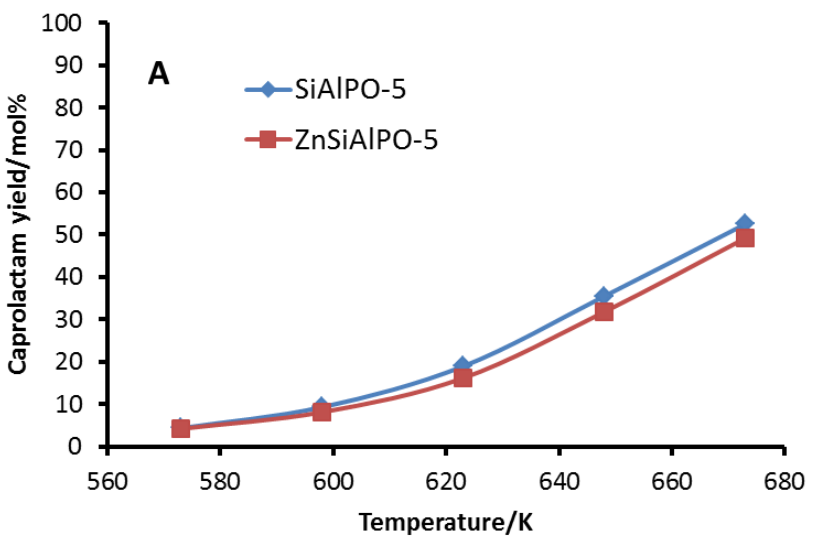

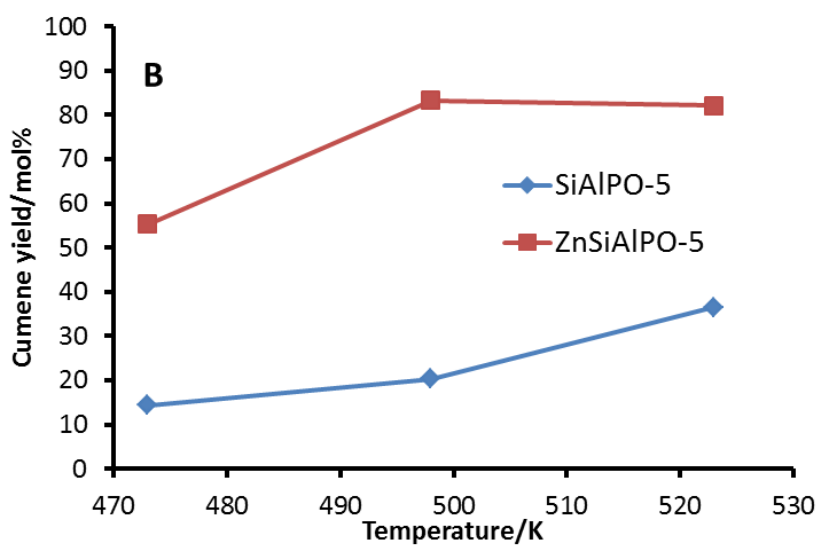

${ }_{25}$ Figure 8 Activity of Si-containing catalysts on the vapour-phase Beckmann Rearrangement (A) and isopropylation of benzene (B). Conditions: A) WHSV $3.3 \mathrm{hr}^{-1}$, Helium carrier gas flow $20 \mathrm{ml} / \mathrm{min}, 0.3 \mathrm{~g}$ of catalyst, liquid feed $300 \mathrm{~g} / \mathrm{l}$ of cyclohexanone oxime in methanol, temperature as shown. B) WHSV of $3.5 \mathrm{hr}^{-1}$, Helium carrier gas of 10

$30 \mathrm{ml} / \mathrm{min}$, feed 6:1 mole ratio of benzene:isopropanol, temperature as shown.

\section{Conclusion}

The ability to succinctly modify acid-site strength, to aid the design and engineering of nanoporous architectures for specific 35 catalytic applications, by the incorporation of $\mathrm{Zn}^{2+}$ ions, has been demonstrated through a combination of in situ FTIR spectroscopy and detailed complementary catalysis results. The versatility of this design approach has been suitably illustrated with contrasting examples of acid-catalysed transformations (requiring diverse 40 active sites), in which the pairing of inactive substituents has created an active solid-acid catalyst. The findings from this study clearly demonstrate that opportune modulation of the strength of the Brønsted active sites, by insertion of $\mathrm{Zn}$, is fundamental to regulating the activity and selectivity of the catalyst for a 45 particular catalytic transformation. Furthermore, the distinctive influence of extra-framework zinc ions in selectively reducing the acid-strength of magnesium prompted an increased effiency for the Beckmann rearrangement of cyclohexanone oxime; whilst concomitantly improving the cumene yield in the isopropylation 50 of benzene, by increasing the acid-strength of the silicon sites. The structure-property correlations that have been substantiated in this study affirm the importance of the design strategy in the creation and modulation of solid-acid active centres, that can impart selectivity control, in acid-catalysed hydrocarbon 55 transformations, offering adequate scope for the rational design of active sites for targeted acid-catalysed reactions.

\section{Notes and references}

${ }^{a}$ School of Chemistry, University of Southampton, Highfield, Southampton, SO17 1BJ, UK.Fax: 02380 593781; Tel:02380592144; E60 mail:R.Raja@soton.ac.uk

${ }^{b}$ Dipartimento di Scienze e Innovazione Tecnologica, Centro Interdisciplinare Nano-SiSTeMI, Università del Piemonte Orientale, via T. Michel 11, I-15100, Alessandria, Italy.

'Diparmento di Chimica e NIS-Centro di Eccellenza, Universita' di ${ }_{65}$ Torino, V. P. Giuria 7 - 10125 Torino, Italy.

We wish to dedicate this article to celebrate the retirement of Professor Salvatore Coluccia. He has been a real source of inspiration for this study and the collaborations outlined herein have contributed to the 
success of the British-Italian partnership program involving the Universities of Southampton and Torino.

$5 \uparrow$ Electronic Supplementary Information (ESI) available including experimental details, ICP data, XRD patterns, probe-based FTIR spectra and in-depth catalysis data. See DOI: 10.1039/b000000x/

1 J. M. Thomas, R. Raja and D. W. Lewis, Angew. Chem. Int. Ed., 2005, 44, 6456.

2 J. M. Thomas and R. Raja, Annu. Rev. Mater. Res., 2005, 35, 315.

3 R. Raja, Top. Catal., 2009, 52, 322.

4 R. M. Leithall, V. N. Shetti, S. Maurelli, M. Chiesa, E. Gianotti and R. Raja, J. Am. Chem. Soc., 2013, 135, 2915.

5 E. Gianotti, V. N. Shetti, M. Manzoli, J. A. L. Blaine, W. C. Pearl Jr., R. D. Adams, S. Coluccia and R. Raja, Chem. Eur. J., 2010, 16, 8202.

6 T. Maschmeyer, F. Rey, G. Sankar and J. M. Thomas, Nature, 1995, 378, 159.

7 A. Thangaraj, R. Kumar and P. Ratsnamay, J. Catal., 1991, 131, 294.

8 A. T. Bell, Science, 2003, 299, 1688.

9 L. Li, G. D. Li, C. Yan, X. Y. Mu, X. L. Pan, X. X. Zou, K. X. Wang and J. S. Chen, Angew. Chem. Int. Ed., 2011, 36, 8449.

10 J. Lee, O. K. Farha, J. Roberts, K. A. Scheidt, S. T. Nguyen and J. T. Hupp, Chem. Soc. Rev., 2009, 38, 1450.

11 L. Kesavan, R. Tiruvalam, M. H. Ab Rahim, M. I. bin Saiman, D. I. Enache, R. L. Jenkins, N. Dimitratos, J. A. Lopez-Sanchez, S. H. Taylor, D. W. Knight, C. J. Kiely and G. J. Hutchings, Science, 2011, 331, 195.

12 R. D. Adams and B. Captain, Angew. Chem. Int. Ed., 2008, 47, 252.

13 A. B. Hungaria, R. Raja, R. D. Adams, B. Captain, J. M. Thomas, P. A. Midgley, V. Golovko and B. F. G. Johnson, Angew. Chem. Int. Ed., 2006, 45, 4782.

14 A. J. Paterson, M. Potter, E. Gianotti and R. Raja, Chem. Commun., 2011, 47, 517.

15 M. E. Potter, A. J. Paterson and R. Raja, ACS Catal., 2012, 2, 2446.

16 B. Weckhuysen, Chem. Commun., 2002, $2,91$.

17 O. V. Safonova, M. Tromp, J. A. van Bokhoven, F. M. F. de Groot, J. Evans and P. Glatzel, J. Phys. Chem. B, 2006, 110, 16162.

18 R. Schlogl and A. Hamid, Angew. Chem. Int. Ed., 2004, 43, 1628 .

19 P. A. Barrett, G. Sankar, C. R. A. Catlow and J. M. Thomas, J. Phys. Chem., 1996, 100, 8977.

20 L. Marchese, T. Maschmeyer, E. Gianotti, S. Coluccia and J. M. Thomas, 1997, 101, 8836.

21 J. A. Lercher, C. Grundling and G. Eder Mirth, Catal. Today, 1996, 27, 353.

22 D. Vitry, Y. Morikawa, J. L. Dubois and W. Ueda, Appl. Catal. A. Gen., 2003, 251, 411.

23 J. M. Thomas and R. Raja, Proc. Natl. Acad. Sci., 2005, 102, 13732.

92, 3965 .

25 C. Zenonos, G. Sankar, F. Cora, D. W. Lewis, Q. A. Pankhurst, C. R. A. Catlow and J. M. Thomas, Phys. Chem. Chem. Phys., 2002, 4, 5421.

65

26 L. Zhou, J. Xu, C. Chen, F. Wang and X. Li, J. Porous Mater., 2008, 15, 7.

27 N. Dimitratos, J. A. Lopez-Sanchez, J. M. Anthonykutty, G. Brett, A. F. Carley, R. C. Tiruvalum, A. A. Herzing, C. J. Kiely, D. W. Knight, G. J. Hutchings, Phys. Chem. Chem. Phys., 2009, 11, 4952.

28 L. Sordelli, R. Psaro, G. Vlaic, A. Cepparo, S. Recchia, C. Dossi, A. Fusi and R. Zanoni, J. Catal., 1999, 182 186.

29 US Pat., US20100249476A1, 2010.

30 J. Silvestre-Albero, J. C. Serrano-Ruiz, A. SepulvedaEscribano and F. Rodriguez-Reinoso, Appl. Catal. A. Gen., 2005, 292, 244.

31 A. B. Fernandez, M. Boronat, T. Blasco ad A. Corma, Angew. Chem. Int. Ed., 2005, 44, 2370.

32 I. Lezcano-Gonzales, A. Vidal-Moya, M. Boronat, T. Blasco, A. Corma, Phys. Chem. Chem. Phys., 2010, 12 , 6396.

33 S. K. Saha, S. B. Waghmode, H. Maekawa, K. Koruma Y. Kubota, Y. Sugi, Y. Oumi and T. Sano, Micro. Meso. Mater., 2005, 81, 289.

34 L. Marchese, J. Chen, J.M. Thomas, S. Coluccia and A. Zecchina, J. Phys. Chem., 1994, 98, 13350.

35 G.E. Ewing, J. Chem. Phys., 1962, 37, 2250.

36 E. Gianotti, V. Dellarocca, E.C. Oliveira, S. Coluccia, H.O. Pastore and L. Marchese, Stud. Surf. Sci. Catal. 2002, 142, 1419.

37 E. Gianotti, C. Bisio, L. Marchese, M. Guidotti, N. Ravasio, R. Psaro and S. Coluccia, J. Phys. Chem. C, 2007, 111, 5083.

38 S. Bordiga, C. Lamberti, F. Geobaldo, A. Zecchina, G. Turnes Palomino and A. Zecchina, Langmuir, 1995, 11, 527.

39 O. Cairon, T. Chevreau and J-C. Lavalley, J. Chem. Soc. Faraday Trans., 1988, 94, 3039.

40 K. G. V. A. Martins, G. Berlier, C. Bisio, S. Coluccia, H.O. Pastore and L. Marchese, J. Phys. Chem. C, 2008, 112, 7193

41 K. Chakarova and K. Hadjiivanov, J. Phys. Chem. C, 2011, 115, 4806.

42 K.I.Hadjiivanov and G.N. Vassilov, Adv. Catal., 2002, 47, 307.

43 C. Lahousse, A. Aboulayt, F. Mauge', J. Bachelier and J.C. Lavalley, J. Mol. Catal. 1993, 84, 283.

44 P.A. Jacobs and C.F. Heylen, J. Catal., 1974, 34, 267.

45 L. Oliviero, A. Vimont, J-C. Lavalley, F.Romero Sarria, M. Gaillard and F. Mauge', Phys. Chem. Chem. Phys., 2005, 7, 1861. 